\title{
The Role of Thymidylate Synthase in Pemetrexed-Resistant Malignant Pleural Mesothelioma Cells
}

\author{
Tohru Obata*, Motohiro Tanaka, Yuka Suzuki, Takuma Sasaki
}

Laboratory of Bioorganic Chemistry, School of Pharmacy, Aichi Gakuin University, Nagoya, Japan.

Email: " tobata@dpc.agu.ac.jp

Received May $15^{\text {th }}, 2013$; revised June $18^{\text {th }}, 2013$; accepted June $25^{\text {th }}, 2013$

Copyright (C) 2013 Tohru Obata et al. This is an open access article distributed under the Creative Commons Attribution License, which permits unrestricted use, distribution, and reproduction in any medium, provided the original work is properly cited.

\begin{abstract}
We established new pemetrexed-resistant cells originating from malignant pleural mesothelioma MSTO-211H cells to clarify the mechanism involved in pemetrexed resistance in malignant pleural mesothelioma. In the pemetrexed-resistant cells, only thymidylate synthase (TYMS) mRNA was overexpressed among other well-known molecular targets and chemosensitivity determinants of pemetrexed, and the role of the TYMS gene was ascertained by artificial regulation induced by specific siRNA. Silencing the TYMS expression partially restored the cytotoxicity of pemetrexed. The resistant cells did not display other gene alterations related to folate metabolism. We conclude that the primary mechanism imparting resistance to these cells is specific up-regulation of TYMS function. Further, the TYMS gene may serve as a useful biomarker for the prediction of pemetrexed chemosensitivity in patients with malignant pleural mesothelioma. We also investigated the efficacy of 1-(3-C-ethynyl- $\beta$-D-ribo-pentofuranosyl)cytosine (ECyd) in overcoming pemetrexed resistance; this compound is presently undergoing clinical trials in the USA as TAS-106. ECyd had a similar antitumor effect on the resistant cells as that on the parental cells. In the clinical treatment of malignant pleural mesothelioma, ECyd promises to emerge as a novel drug.
\end{abstract}

Keywords: Malignant Pleural Mesothelioma; Thymidylate Synthase; Pemetrexed; ECyd

\section{Introduction}

Malignant pleural mesothelioma is considered to be caused by previous exposure to asbestos fibers [1-3]. Regardless of the stage at diagnosis, it is generally viewed as a treatment-resistant tumor having poor prognosis [4] owing to serious difficulties, including the availability of effective drugs. Until recently, although no chemotherapeutic agent was effective against malignant pleural mesothelioma, in 2004, the Food and Drug Administration (FDA) approved administration of pemetrexed in combination with cisplatin for the treatment of patients whose condition is unresectable or who are otherwise not candidates for curative surgery [5]. Pemetrexed is an antifolate drug that targets some folate enzymes [5,6]; this drug is initially transported into the cytoplasm by reduced folate carrier (RFC) and other transporters and then metabolized to a polyglutamated form by folypoly-gamma-glutamate synthetase (FPGS). Polyglutamation increases cellular reten-

${ }^{*}$ Corresponding author. tion and confers an affinity for some enzymes involved in folate metabolism. Pemetrexed and its polyglutamated derivatives inhibit thymidylate synthase (TYMS), dihydrofolate reductase (DHFR), and glycinamide ribonucleotide transformylase (GARFT), all of which are involved in the de novo biosynthesis of thymidine and purine nucleotides. Antimetabolite agents, including pemetrexed, induce an imbalance in the cellular nucleotide pool and inhibit nucleic acid biosynthesis that results in arresting the proliferation of tumor cells and inducing cell death [5-7].

In pemetrexed-resistant cells, TYMS overexpression is one of the major factors leading to resistance [8] and the regulation of DHFR, RFC, and FPGS expression is associated with acquired resistance to pemetrexed $[8,9]$. These resistance mechanisms have been investigated in the colon [10], breast [11], gastric [12], small cell lung cancer [13], non-small cell lung cancer [14], and leukemia cell lines [15]. These tumor types had not been recognized by FDA. In this study, we have newly established peme- 
trexed-resistant malignant pleural mesothelioma cells from MSTO-211H cells and studied its resistant mechanisms against pemetrexed. Further, we also examined the efficacy of 1-(3-C-ethynyl- $\beta$-D-ribo-pentofuranosyl)cytosine (ECyd) against malignant pleural mesothelioma in overcoming the established resistance.

\section{Materials and Methods}

\subsection{Drugs and Tumor Cells}

1-(3-C-Ethynyl- $\beta$-D-ribo-pentofuranosyl)cytosine (ECyd, TAS-106) was provided by TAIHO Pharmaceutical (Tokyo, Japan), while pemetrexed was purchased from Toronto Research Chemicals (North York, Canada). Human mesothelioma MSTO-211H cells were purchased from the American Type Culture Collection (ATCC, Manassas, VA, USA). The resistant cell line (H/pemetrexed) was established by a stepwise drug increase method. Both parental and resistant cell lines were maintained at $37^{\circ} \mathrm{C}$ and $5 \% \mathrm{CO}_{2}$ in RPMI-1640 medium supplemented with $10 \%$ heat-inactivated fetal bovine serum (FBS) and 1\% penicillin-streptomycin (Life Technologies, Carlsbad, CA, USA).

\subsection{Drug Sensitivity Test}

The growth-inhibitory effects of the drugs on human tumor cells were examined using a colorimetric assay involving 3-(4,5-dimethylthiazol-2-yl)-2,5-diphenyltetrazolium bromide (MTT). Briefly, 190- $\mu$ l aliquots of an exponentially growing cell suspension (1000 cells/190 $\mu \mathrm{l} /$ well) were incubated with $10 \mu \mathrm{l}$ of varying concentrations of drugs. After exposure to the drugs for $48-72 \mathrm{~h}$, $20 \mu \mathrm{l}$ of MTT solution ( $3 \mathrm{mg} / \mathrm{ml}$ ) was added to each well and the cell cultures were incubated at $37^{\circ} \mathrm{C}$ for $4 \mathrm{~h}$. After removal of the medium, the formed formazan was dissolved in $200 \mu \mathrm{l}$ of dimethyl sulfoxide. The absorbance of each well was measured at $570 \mathrm{~nm}$ with an immunoreader (MTP-800AFC, CORONA Electric, Hitachinaka, Japan), and the inhibition ratio (IR) was calculated using the following formula: IR $(\%)=(1-\mathrm{T} / \mathrm{C}) \times 100$, where $\mathrm{C}$ is the mean of optical densities of the control group and $\mathrm{T}$ of the treatment group. The $\mathrm{IC}_{50}$ value was defined as the concentration of the drug needed to effect a $50 \%$ reduction in growth relative to the control. $\mathrm{The}^{\mathrm{IC}_{50}}$ value was determined by a graphical correlation of the doseresponse curve with at least three drug concentration points.

\section{3. siRNA Transfection}

All small-interfering RNAs (siRNAs) were purchased from Stealth RNAi (Life Technologies). The sequences of three siRNAs (TYMS-1 to -3) targeting TYMS gene (NM_001071) were

\section{5'-GCTGTGGTTTATCAAGGGATCCACA-3' \\ (403 - 427), \\ 5'-GGGAGATGCACATATTTACCTGAAT-3' \\ (895 - 919), and \\ 5'-CAGAGATATGGAATCAGATTATTCA-3'}

(577 - 919), respectively. Stealth RNAi glyceraldehyde3-phosphate dehydrogenase (GAPDH) Positive Control (Life Technologies) and Stealth RNAi Negative Control (Low GC Duplex \#2; Life Technologies) were served as a control. Upon preincubation at $37^{\circ} \mathrm{C}$ for $24 \mathrm{~h}$, cells in 35-mm dishes were transfected with 250 pmol siRNA using lipofectamine 2000 (Life Technologies) following the manufacturer's protocol. Cells were treated with the transfection agent/siRNA complex for $24 \mathrm{~h}$ and subjected to further analysis.

\subsection{Quantization of mRNA Expression}

The cells were washed with PBS (-) and then total RNA was extracted using ISOGEN (Nippon Gene, Osaka, Japan) according to the manufacturer's instructions. The concentration of the total extracted RNA was determined by measuring the OD at $260 \mathrm{~nm}$, and the RNA was diluted to $200 \mu \mathrm{g} / \mathrm{ml}$. First strand cDNA synthesis was carried out using $2 \mu \mathrm{g}$ of total RNA, 5 pmol oligo (dT) 12 18 (GE Healthcare, Buckinghamshire, UK) and ReveTraAce (TOYOBO, Osaka, Japan) at $42^{\circ} \mathrm{C}$ for $90 \mathrm{~min}$. cDNA prepared by the reverse transcription reaction was subjected to PCR amplification in a Thermal Cycler Dice Real-Time System (Takara Bio, Shiga) with SYBR Green PCR Master Mix (Takara Bio, Japan) using specific primers (Table 1). The expression of the target genes was standardized using the regular housekeeping genes [ribosomal protein large P2 (RPLP2), ribosomal protein S18 (RPS18), phosphoglycerate kinase 1 (PGK1), and beta-actin $(A C T B)]$, and the relative expression levels were quantified by using the $2^{-\Delta \Delta \mathrm{CT}}$ method.

\subsection{Establishment of TYMS-Overexpressing Tumor Cell Line}

Cells were transfected with the TYMS gene to clarify and understand the effect of the drugs on TYMS function. Human TYMS was overexpressed by PCR amplification of the full coding sequence of human TYMS cDNA using the sense primer 5'-ATGCCTGTGGCCGGCTCGGA-3' and the antisense primer

5'-ATATCCTTCGAGCTCCTTTG-3'. The TYMS cDNA was cloned into the pEF6/V5-His TOPO vector (Life Technologies) to construct pEF6/TYMS. This plasmid was transfected into the MSTO-211H cells by using the FuGENE 6 transfection reagent (Roche Diagnostics, Indianapolis, IN, USA) according to the manufacturer's instructions. The transfectants were grown in a cultured medium containing $500 \mu \mathrm{g} / \mathrm{ml}$ blasticidin S. Blasticidin- 
Table 1. Primers used in real-time PCR.

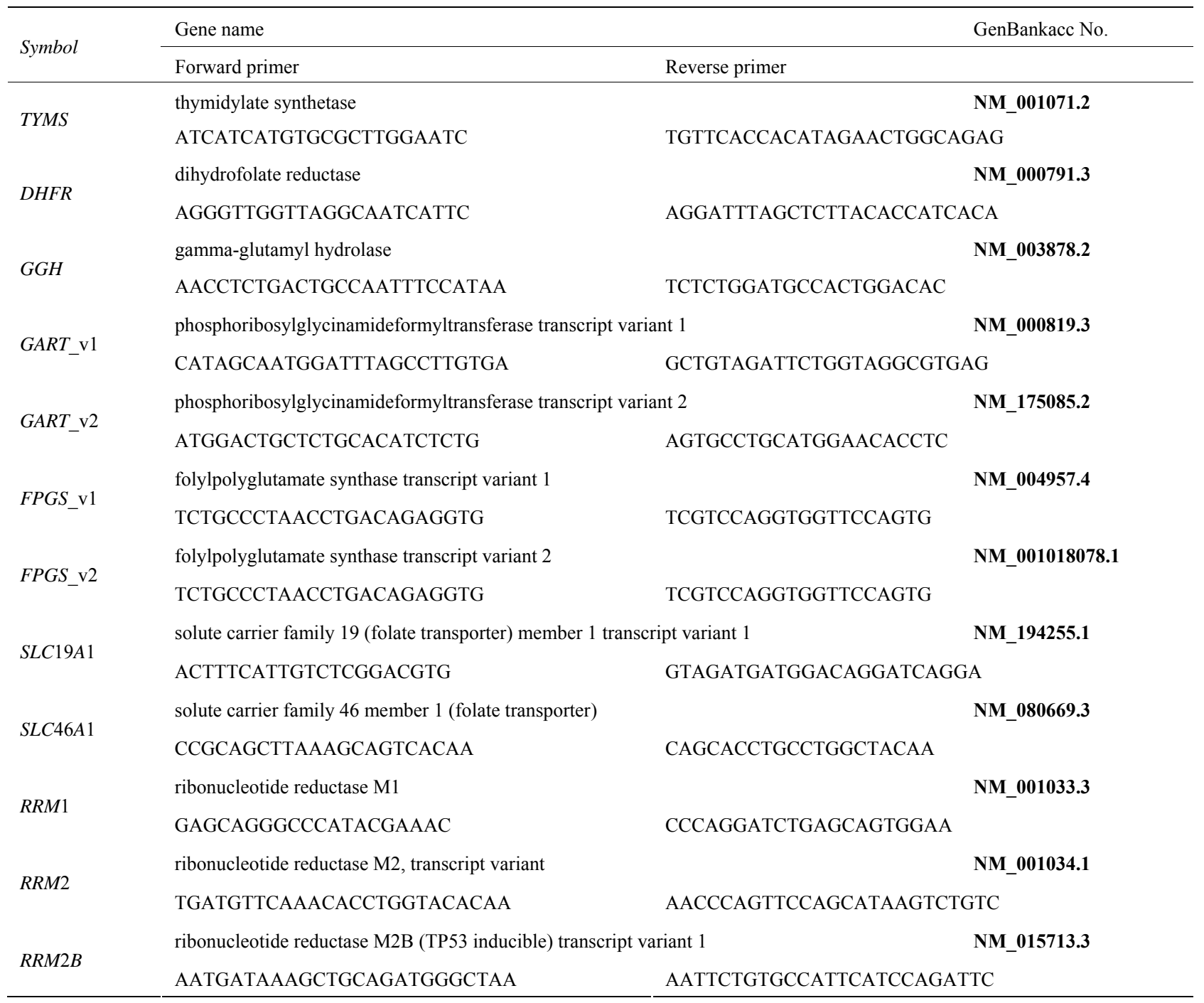

resistant cells were isolated and designated as MSTO/ TYMS. The empty vector pEF6 was also transfected into the MSTO- $211 \mathrm{H}$ cells to generate control cells, which were designated as MSTO/Mock.

\subsection{Western Blot Analysis for Protein Expression of Human TYMS}

Cell lysates were prepared in CelLytic-M reagent (Sigma-Aldrich, St. Louis, MO, USA) containing 10\% Protease Inhibitor Cocktail (Sigma-Aldrich). Protein samples were mixed with a loading buffer [50 mMTris$\mathrm{HCl}(\mathrm{pH} 6.5), 10 \%$ glycerol, $2 \%$ sodium dodecyl sulfate (SDS), $0.1 \%$ bromophenol blue, and $40 \mathrm{mM}$ dithiothrei tol] and electrophoresed on a $10 \%-20 \%$ gradient SDSpoly-acrylamide gel (mini-quick gel, Anatech, Tokyo, Japan) after which the proteins were transferred to a polyvinylidene difluoride membrane filter (Immobilon; Millipore, Bedford, MA, USA). The membrane was blocked in TBS-Tween containing 5\% blocking agent (GE Healthcare) for $1 \mathrm{~h}$ and then probed with the mouse monoclonal antibody to TYMS (ab3145, Abcam, Cambridge, UK) or with rabbit polyclonal antibody to B-actin (ab8227, Abcam) for $2 \mathrm{~h}$ at room temperature. Horseradish peroxidase-conjugated anti-mouse IgG antibody or anti-rabbit IgG antibody (GE Healthcare) was used for the detection with enhanced chemiluminescence detection reagent (GE Healthcare). Chemiluminescence was detected by LAS-3000 (Fuji Film, Tokyo, Japan).

\section{Results}

\subsection{Establishment of Pemetrexed-Resistant Cells Originating from Malignant Pleural Mesothelioma}

The MSTO-211H cells were initially cultured with $1 \mathrm{nM}$ pemetrexed. Incremental increase in the concentration of 
pemetrexed during logarithmic cell growth allowed the determination of maximum growth rate, and eventually, the cells were grown in the culture medium containing $0.1 \mu \mathrm{M}$ pemetrexed. The dose-response curves for pemetrexed exposed to parental MSTO- $211 \mathrm{H}$ cells and resistant cells (H/pemetrexed) are presented in Figure 1. The $\mathrm{IC}_{50}$ value of the $\mathrm{H} /$ pemetrexed cells was estimated to be over 100 times that of the parental cells.

\subsection{Comparison of the Expression of Related Genes during Folate Metabolism}

Expression of several typical genes related to pemetrexed chemosensitivity was measured by real-time PCR, and the TYMS mRNA expression $(32.9 \pm 9.7$ times $)$ was found to be significantly increased in $\mathrm{H} /$ pemetrexed cells (Figure 2(a)). The expression of DHFR, gammaglutamyl hydrolase $(G G H)$, ribonucleotide reductase M1 $(R R M 1)$, and ribonucleotide reductase $\mathrm{M} 2 \mathrm{~B}(R R M 2 B)$ genes also increased slightly in the $\mathrm{H} /$ pemetrexed cells (3.2 to 4.6-fold, statistically insignificant). On the other hand, the expression of solute carrier family 19 member (SLC19A) mRNA, which is one of the folate transporters, was significantly reduced compared to the parental cells $(0.53 \pm 0.05$ times $)$. Overexpression of the TYMS protein was also confirmed by western blot analysis (Figure 2(b)).

\subsection{Drug Sensitivity to Assess the Effect of Transfection with siRNA Targeted to the TYMS Gene}

Intracellular mRNA expression can be downregulated by specific siRNA. All three TYMS siRNAs, which targeted independent sequences within the TYMS coding region, effectively induced downregulation of the intracellular

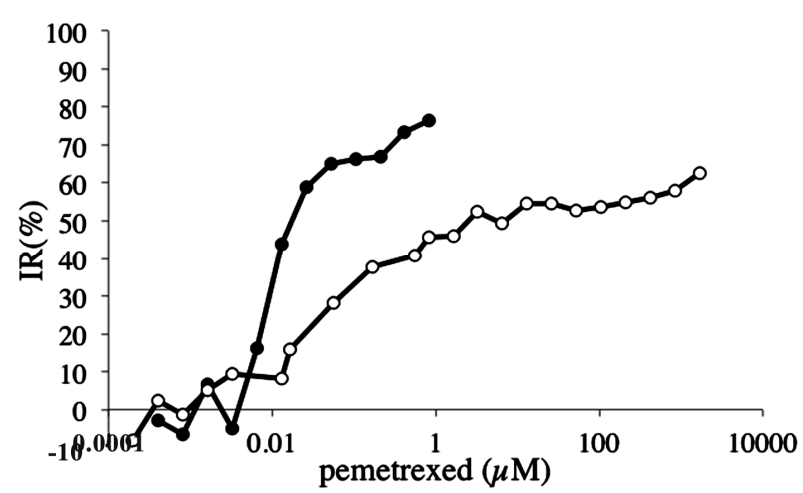

Figure 1. Chemosensitivity in pemetrexed-resistant cells originating from malignant pleural mesothelioma. Doseresponse curves for pemetrexed in MSTO-211H cells (closed circles) and in $\mathbf{H} /$ pemetrexed cells (open circles). Continuous exposure to variable concentrations of pemetrexed applied for $72 \mathrm{~h}$. Each point was plotted as an average of easily three independent experiments.
TYMS mRNA expression by over $80 \%$ in $\mathrm{H} /$ pemetrexed and MSTO-211H cells (Figure 3). Control siRNA, targeted towards the GAPDH gene, also specifically downregulated the expression of GAPDH mRNA. Each siRNA had an effect exclusively on the expression of its specific target gene in both the cells. The cells treated with siRNA were exposed to varying concentrations of pemetrexed for $48 \mathrm{~h}$ after which their chemosensitivity was evaluated. Pemetrexed was applied at two varying concentrations, as each cell line possessed considerably

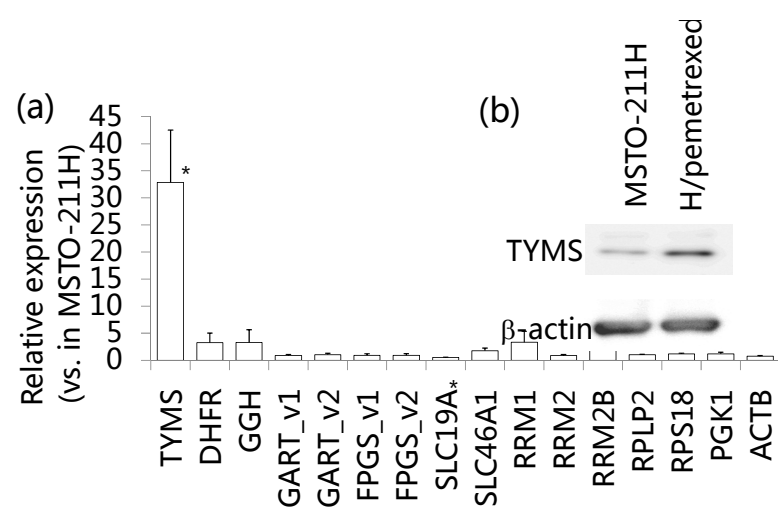

Figure 2. Comparison of anti-folate metabolism genes between parental and resistant cells. (a) mRNA expression was evaluated by real-time RT-PCR using the $\Delta \Delta C T$ method normalized by housekeeping genes. "shows significant difference from parental MSTO-211H cells by the t-test (p< 0.01); (b) The level of TYMS protein in MSTO$211 \mathrm{H}$ cells and $\mathrm{H} /$ pemetrexed cells as detected by the western blot analysis.

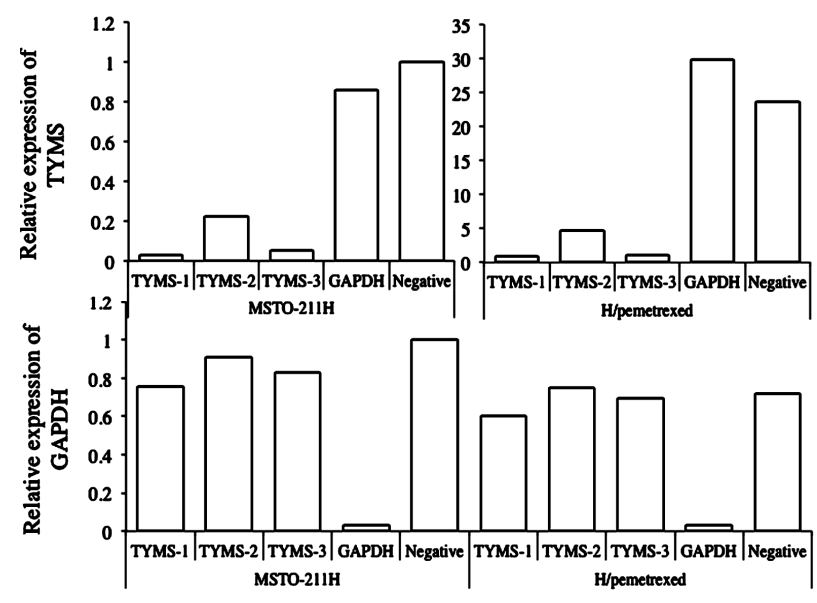

Figure 3. Quantization of mRNA expression in siRNA treated MSTO-211H and H/pemetrexed cells. Three TYMS siRNAs were targeted for different TYMS gene sequences and transfected into MSTO-211H and H/pemetrexed cells. GAPDH and negative siRNA were used as controls. mRNA expression was evaluated by quantitative real-time PCR. Upper and lower graphs represent the expression of TYMS and GAPDH mRNAs, respectively. Each expression fold was calculated from the ratio of MSTO-211H cells treated with negative siRNA. 
different chemosensitivity to pemetrexed. Treating the parental cells with two siRNAs targeting TYMS (TYMS -2 and -3 ) enhanced the antitumor activity (27 and 15\%) at $0.007 \mu \mathrm{M}$ pemetrexed (Figure 4). Moreover, the effect of TYMS siRNA was confirmed even in $\mathrm{H} /$ pemetrexed cells that had overexpressed TYMS mRNA. In both cell lines, siRNAs targeted at TYMS increased the cytotoxicity of pemetrexed.

\subsection{Reduction of Drug Sensitivity in Transfected Cells with TYMS Expression Vector}

In order to determine whether TYMS gene expression was affected by pemetrexed sensitivity, the parental cells were transfected with a TYMS expression vector. The TYMS expression vector (pEF6/TYMS) was constructed from $\mathrm{pEF} 6 / \mathrm{V} 5$-His TOPO vector according to the given protocol, and the constructed vector was transfected into the MSTO-211H cells to establish stable TYMS overexpressed cells (MSTO/TYMS). The expression of TYMS mRNA in these cells was confirmed by real-time PCR (Figure 5) and the expression levels in the MSTO/ TYMS cells against parental MSTO-211H cells $(21.9 \pm$ 3.9 fold) was similar to that of $\mathrm{H} /$ pemetrexed cells. Pemetrexed chemosensitivity in the established MSTO/ TYMS cells was investigated (Figure 6) and found to be reduced, having $\mathrm{IC}_{50}$ values higher than those of the parental or mock cells. Furthermore, a cross-resistance to methotrexate (MTX) was also observed.

\subsection{Antitumor Activity of ECyd against Pemetrexed-Resistant Cells}

The cytotoxicity of other antimetabolic drugs was inves tigated for overcoming the high pemetrexed-resistance in H/pemetrexed cells (Table 2). ECyd showed similar antitumor effect against both parental and resistant cells.

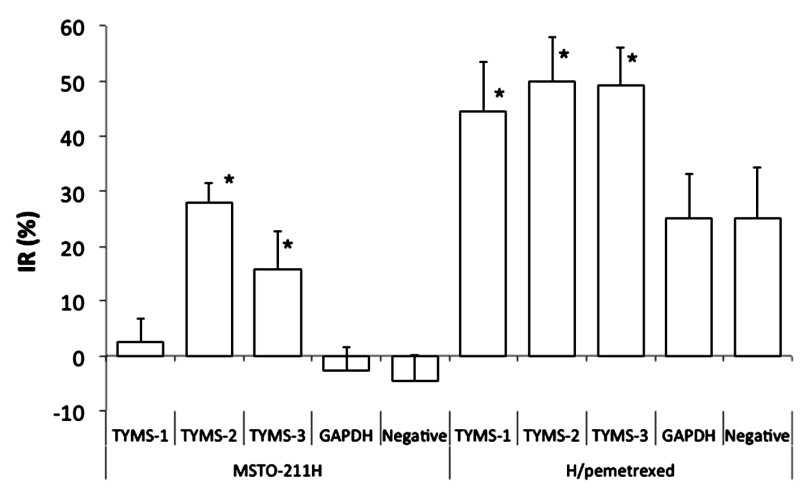

Figure 4. Effect of TYMS siRNA on cytotoxicity of pemetrexed in MSTO-211H and H/pemetrexed cells. MSTO$211 \mathrm{H}$ and $\mathrm{H} /$ pemetrexed cells were exposed to 0.007 and $840 \mu \mathrm{M}$ of pemetrexed for $48 \mathrm{~h}$ after transfection with specific siRNA. "shows significant difference from negative siRNA treated cells by the t-test $(p<0.01)$.

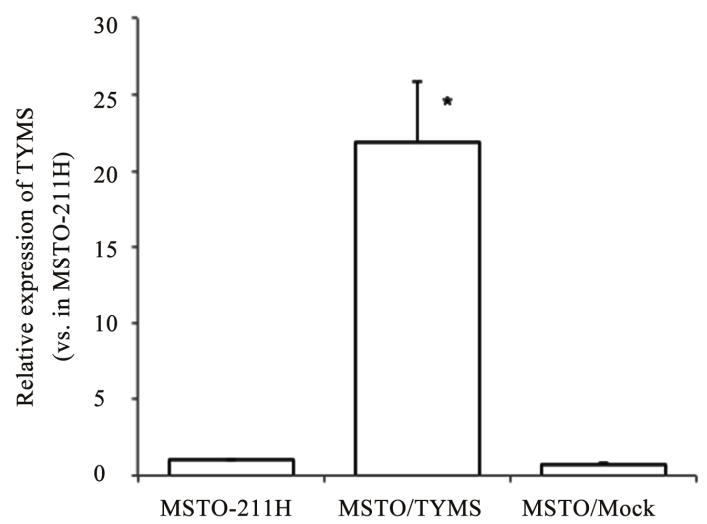

Figure 5. mRNA expression in MSTO-211H cells transfected with TYMS expression vector. MSTO-211H cells transfected with TYMS expression vector were established by a selection agent over a few weeks. The mRNA expression was evaluated by quantitative real-time PCR. Each expression fold was calculated by the ratio against MSTO$211 \mathrm{H}$ cells. "shows significant difference from parental MSTO-211H cells by the t-test $(p<0.01)$.

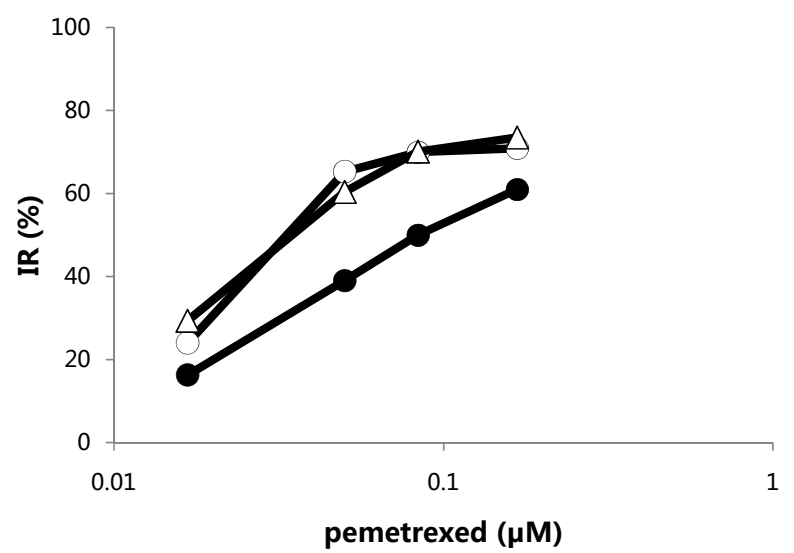

(a)

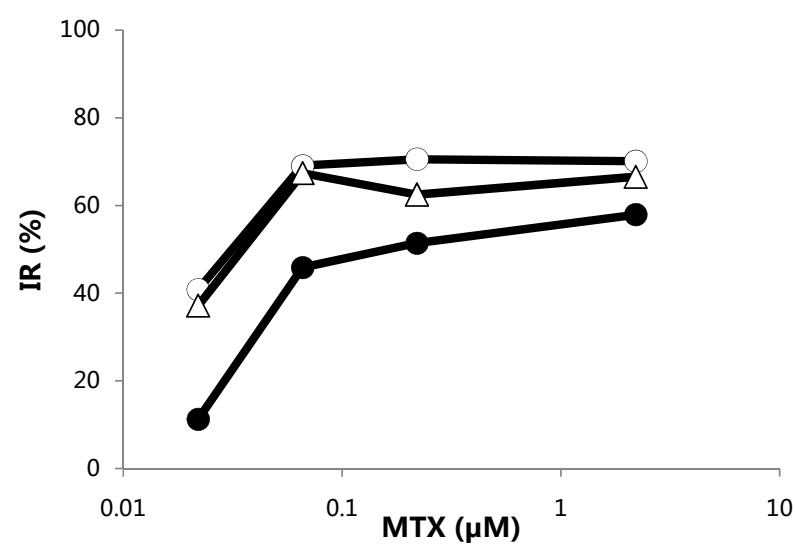

(b)

Figure 6. Inhibition of antitumor effect by transfection with TYMS expression vector. The chemosensitivity of pemetrexed and MTX is shown in MSTO-211H cells (open circles), MSTO/TYMS cells (closed circles) and MSTO/Mock cells (open triangles). 
Table 2. Cytotoxicity profiles of antimetabolic drugs in H/pemetrexed cells.

\begin{tabular}{cccc}
\hline \multirow{2}{*}{ Drug } & \multicolumn{2}{c}{$\mathrm{IC}_{50}(\mu \mathrm{M})$} & $\begin{array}{c}\text { Resistant } \\
\text { index }^{*}\end{array}$ \\
\cline { 2 - 3 } Pemetrexed & 0.067 & 8.70 & 130 \\
MTX & 0.012 & 0.066 & 5.67 \\
5FU & 7.46 & 13.7 & 1.84 \\
ECyd & 0.029 & 0.012 & 0.43 \\
\hline
\end{tabular}

${ }^{*}$ Resistance index was calculated as a ratio of $\mathrm{IC}_{50}$ for resistant cells to $\mathrm{IC}_{50}$ for parental cells.

Since MTX had a similar mechanism as pemetrexed, partial cross-resistance was observed in $\mathrm{H} /$ pemetrexed cells. Additionally, 5FU had an equivalent antitumor effect, which was revealed even in $\mathrm{H} /$ pemetrexed cells.

\section{Discussion}

Although several pemetrexed-resistant cells originating from colon, breast, gastric and non-small cell lung cancers and especially leukemia cell lines have already been reported, there is no report of pemetrexed-resistance cells from malignant pleural mesothelioma cell lines. Recently, pemetrexed has been clinically approved against malignnant pleural mesothelioma, but continuous and repeated treatment can result in resistance against permetrexed in the future. We attempted to establish pemetrexed-resistant cells originating from some malignant pleural mesothelioma cell lines in order to clarify the mechanism underlying pemetrexed resistance in mesothelioma. Among the cell lines tested, only the MSTO- $211 \mathrm{H}$ cells could acquire resistance against pemetrexed. Treatment of MSTO-211H cells with pemetrexed induced higher expression of TYMS mRNA compared to other mesothelioma cell lines, such as NCI-H2452 and ACC-MESO-1. This characteristic nature of MSTO-211H cells may contribute to the acquisition of high resistance against pemetrexed.

Detailed pemetrexed-resistant mechanisms in $\mathrm{H} / \mathrm{pe}-$ metrexed cells were investigated by analyzing mRNA expression, which were closely related to folate metabolism. Remarkably, TYMS mRNA was expressed in H/ pemetrexed cells, and the expression of some genes, including DHFR, GGH, and $R R$, increased slightly. The main reason for pemetrexed-resistance in $\mathrm{H} /$ pemetrexed cells was considered to be an up-regulation of the TYMS function. However, contribution of the up-regulation of $D H F R, G G H$, and RR genes in the elevated pemetrexedresistance could not be completely ruled out. The expression of SLC19A, a folate-transporter gene, decreased significantly and there appeared to be a weak relationship with pemetrexed resistance. Additionally, pemetrexed may be specifically transported intracellularly via $S L C$ $19 A 1$ and not SLC46A1.

Single nucleotide polymorphism (SNP) in the 5'-UTR of the TYMS gene is well known, and the tandem repeat is related to the chemosensitivity of 5FU [16-19]. The overexpression of TYMS mRNA could not be attributed to SNP in the 5'-UTR, since the genomic polymorphisms (3R type) among the parental and resistant cells were not altered. Moreover, since the $E 2 F$ of a transcription factor resided within the promoter region of the TYMS gene, the mRNA expression of the E2F family was investigated. We observed that the expression of E2F family mRNA was not upregulated in the H/pemetrexed cells. Therefore, even though the activation mechanism of the TYMS gene in $\mathrm{H} /$ pemetrexed cells has not yet been identified, the TYMS gene is considered to be an important factor in the acquisition of pemetrexed-resistance.

The TYMS gene was artificially regulated to clarify its involvement in pemetrexed resistance. TYMS down-regulation by specific siRNA partially restored the chemosensitivity for pemetrexed in the parental and $\mathrm{H} /$ pemetrexed cells. However, the effect of TYMS-1 siRNA on pemetrexed chemosensitivity did not appear unexpectedly. Although TYMS-1 siRNA knocked down TYMS mRNA, the chemosensitivity for pemetrexed in only the parental MSTO- $211 \mathrm{H}$ cells were similar to that treated with control siRNA. Since TYMS mRNA expression in MSTO- $211 \mathrm{H}$ cells were steady at a lower level than that in $\mathrm{H} /$ pemetrexed cells, the enhanced cytotoxic effect on being treated with specific siRNA in MSTO- $211 \mathrm{H}$ cells are plausible. On the other hand, MSTO/ TYMS cells, which stably over-expressed TYMS mRNA, tended to be resistant against pemetrexed and MTX. Even though the expression level of TYMS mRNA in MSTO/TYMS cells were over 20 times higher than that in MSTO- $211 \mathrm{H}$ cells, its resistance were weak relative to $\mathrm{H} /$ pemetrexed cells, which similarly over-expressed TYMS mRNA. In H/pemetrexed cells, the expression of other genes, including DHFR and GGH, increased slightly but not in MSTO/ TYMS cells. It was considered that the functions of DHFR and $G G H$ were partially related to acquisition of pemetrexed resistance in the MSTO-211H cells.

High resistance to an antitumor drug is a serious problem in chemotherapy. We have examined the cytotoxicity of some antimetabolic drugs in $\mathrm{H} /$ pemetrexed cells (Table 2) and found that these cells showed cross-resistance to MTX, which had cellular metabolism and targets similar to pemetrexed. Although 5FU targets the same cellular factors as MTX, the chemosensitivity for $5 \mathrm{FU}$ in $\mathrm{H} /$ pemetrexed cells were retained, suggesting that the main target of 5FU in this cell line is not TYMS. It appeared that the pyrimidine salvage pathway was more prominent than in other cell lines. ECyd, having an antitumor mechanism different from pemetrexed, was an 
effective drug even in pemetrexed-resistant cells. However, the mechanism of ECyd involves inhibition of RNA biosynthesis, and ECyd has been considered a superior antitumor nucleoside whose clinical trials are in progress as TAS-106 in USA $[20,21]$. ECyd and pemetrexed belong to the same category as anti-metabolic drugs. However, their metabolic and activated pathways differ through the pyrimidine and folate metabolic pathways, respectively, bestowing ECyd with excellent antitumor activity against many solid tumors [22-28]. Even in H/pemetrexed cells, ECyd showed the same antitumor effect as in MSTO-211H cells. Therefore, in the clinical treatment of malignant pleural mesothelioma patients, ECyd may be extremely useful as a promising second line drug. The TYMS gene may be considered as a useful biomarker for predicting pemetrexed chemosensitivity in malignant pleural mesothelioma patients.

\section{REFERENCES}

[1] M. Pistolesi and J. Rusthoven, "Malignant Pleural Mesothelioma: Update, Current Management, and Newer Therapeutic Strategies," Chest, Vol. 126, No. 4, 2004, pp. 1318-1329. doi:10.1378/chest.126.4.1318

[2] M. E. Ramos-Nino, J. R. Testa, D. A. Altomare, H. I. Pass, M. Carbone, M. Bocchetta and B. T. Mossman, "Cellular and Molecular Parameters of Mesothelioma," Journal of Cellular Biochemistry, Vol. 98, No. 4, 2006, pp. 723-734. doi: $10.1002 /$ jcb. 20828

[3] B. W. Robinson and R. A. Lake, "Advances in Malignant Mesothelioma," New England Journal of Medicine, Vol. 353, No. 15, 2005, pp. 1591-1603. doi:10.1056/NEJMra050152

[4] J. P. Steele and A. Klabatsa, "Chemotherapy Options and New Advances in Malignant Pleural Mesothelioma," Annals of Oncology, Vol. 16, No. 3, 2005, pp. 345-351. doi:10.1093/annonc/mdi094

[5] M. Hazarika, R. M. White, J. R. Johnson and R. Pazdur, "FDA Drug Approval Summaries: Pemetrexed (Alimta)," Oncologist, Vol. 9, No. 5, 2004, pp. 482-488.

[6] S. Chattopadhyay, R. G. Moran and I. D. Goldman, "Pemetrexed: Biochemical and Cellular Pharmacology, Mechanisms, and Clinical Applications," Molecular Cancer Therapeutics, Vol. 6, No. 2, 2007, pp. 404-417. doi:10.1158/1535-7163.MCT-06-0343

[7] M. Hazarika, R. M. White Jr., B. P. Booth, Y. C. Wang, D. Y. Ham, C. Y. Liang, A. Rahman, J. V. Gobburu, N. Li, R. Sridhara, D. E. Morse, R. Lostritto, P. Garvey, J. R. Johnson and R. Pazdur, "Pemetrexed in Malignant Pleural Mesothelioma," Clinical Cancer Research, Vol. 11, No. 3, 2005, pp. 982-992.

[8] R. Zhao and I. D. Goldman, "Resistance to Antifolates," Oncogene, Vol. 22, No. 47, 2003, pp. 7431-7457.

[9] N. Hagner and M. Joerger, "Cancer Chemotherapy: Targeting Folic Acid Synthesis," Cancer Management and Research, Vol. 2, 2010, pp. 293-301.

[10] J. Sigmond, H. H. Backus, D. Wouters, O. H. Temmink,
G. Jansen and G. J. Peters, "Induction of Resistance to the Multitargeted Antifolate Pemetrexed (ALIMTA) in WiDr Human Colon Cancer Cells Is Associated with Thymidylate Synthase Overexpression," Biochemical Pharmacology, Vol. 66, No. 3, 2003, pp. 431-438. doi:10.1016/S0006-2952(03)00287-9

[11] D. B. Longley, P. R. Ferguson, J. Boyer, T. Latif, M. Lynch, P. Maxwell, D. P. Harkin and P. G. Johnston, "Characterization of a Thymidylate Synthase (TS)-Inducible Cell Line: A Model System for Studying Sensitivity to TS- and Non-TS-Targeted Chemotherapies," Clinical Cancer Research, Vol. 7, No. 11, 2001, pp. 3533-3539.

[12] J. H. Kim, K. W. Lee, Y. Jung, T. Y. Kim, H. S. Ham, H. S. Jong, K. H. Jung, S. A. Im, T. Y. Kim, N. K. Kim and Y. J. Bang, "Cytotoxic Effects of Pemetrexed in Gastric Cancer Cells," Cancer Science, Vol. 96, No. 6, 2005, pp. 365-371. doi:10.1111/j.1349-7006.2005.00058.x

[13] H. Ozasa, T. Oguri, T. Uemura, M. Miyazaki, K. Maeno, S. Sato and R. Ueda, "Significance of Thymidylate Synthase for Resistance to Pemetrexed in Lung Cancer," Cancer Science, Vol. 101, No. 1, 2010, pp. 161-166. doi:10.1111/j.1349-7006.2009.01358.x

[14] D. Zhang, N. Ochi, N. Takigawa, Y. Tanimoto, Y. Chen, E. Ichihara, K. Hotta, M. Tabata, M. Tanimoto and K. Kiura, "Establishment of Pemetrexed-Resistant Non-Small Cell Lung Cancer Cell Lines," Cancer Letters, Vol. 309, No. 2, 2011, pp. 228-235. doi:10.1016/j.canlet.2011.06.006

[15] Y. Wang, R. Zhao and I. D. Goldman, "Decreased Expression of the Reduced Folate Carrier and Folypolyglutamate Synthetase Is the Basis for Acquired Resistance to the Pemetrexed Antifolate (LY231514) in an L1210 Murine Leukemia Cell Line," Biochemical Pharmacology, Vol. 65, No. 7, 2003, pp. 1163-1170. doi:10.1016/S0006-2952(03)00007-8

[16] Q. Zhang, Y. P. Zhao, Q. Liao, Y. Hu, Q. Xu, L. Zhou and H. Shu, "Associations between Gene Polymorphisms of Thymidylate Synthase with Its Protein Expression and Chemosensitivity to 5-Fluorouracil in Pancreatic Carcinoma Cells," Chinese Medical Journal (English Edition), Vol. 124, No. 2, 2011, pp. 262-267.

[17] K. Kawakami and G. Watanabe, "Identification and Functional Analysis of Single Nucleotide Polymorphism in the Tandem Repeat Sequence of Thymidylate Synthase Gene," Cancer Research, Vol. 63, No. 18, 2003, pp. 6004-6007.

[18] N. Nief, V. Le Morvan and J. Robert, "Involvement of Gene Polymorphisms of Thymidylate Synthase in Gene Expression, Protein Activity and Anticancer Drug Cytotoxicity Using the NCI-60 Panel," European Journal of Cancer, Vol. 43, No. 5, 2007, pp. 955-962. doi:10.1016/j.ejca.2006.12.012

[19] M. Gusella and R. Padrini, "G $>$ C SNP of Thymidylate Synthase with Respect to Colorectal Cancer," Pharmacogenomics, Vol. 8, No. 8, 2007, pp. 985-996. doi: $10.2217 / 14622416.8 .8 .985$

[20] L. A. Hammond-Thelin, M. B. Thomas, M. Iwasaki, J. L. Abbruzzese, Y. Lassere, C. A. Meyers, P. Hoff, J. de Bono, J. Norris, H. Matsushita, A. Mita and E. K. Rowinsky, "Phase I and Pharmacokinetic Study of 3'-C-ethynyl- 
cytidine (TAS-106), an Inhibitor of RNA Polymerase I, II and III, in Patients with Advanced Solid Malignancies," Investigational New Drugs, Vol. 30, No. 1, 2012, pp. 316-326. doi:10.1007/s10637-010-9535-y

[21] B. Friday, Y. Lassere, C. A. Meyers, A. Mita, J. L. Abbruzzese and M. B. Thomas, "A Phase I Study to Determine the Safety and Pharmacokinetics of Intravenous Administration of TAS-106 Once per Week for Three Consecutive Weeks Every 28 Days in Patients with Solid Tumors," Anticancer Research, Vol. 32, No. 5, 2012, pp. 1689-1696.

[22] S. Tabata, M. Tanaka, A. Matsuda, M. Fukushima and T. Sasaki, "Antitumor Effect of a Novel Multifunctional Antitumor Nucleoside, 3'-Ethynylcytidine, on Human Cancers," Oncology Reports, Vol. 3, No. 6, 1996, pp. 10291034.

[23] M. Tanaka, S. Tabata, A. Matsuda, M. Fukushima, K. Eshima and T. Sasaki, "Antitumor Effect and Mechanism of a Novel Multifunctional Nucleoside, 3'-Ethynylnucleoside, on Human Cancers," Gan to Kagaku Ryoho, Vol. 24, No. 4, 1997, pp. 476-482.

[24] S. Tabata, M. Tanaka, Y. Endo, T. Obata, A. Matsuda and T. Sasaki, "Anti-Tumor Mechanisms of 3'-Ethynyluridine and 3'-Ethynylcytidine as RNA Synthesis Inhibitors: De- velopment and Characterization of 3'-Ethynyluridine-Resistant Cells," Cancer Letters, Vol. 116, No. 2, 1997, pp. 225-231. doi:10.1016/S0304-3835(97)00188-2

[25] A. Matsuda, M. Fukushima, Y. Wataya and T. Sasaki, “A New Antitumor Nucleoside, 1-(3-C-ethynyl- $\beta$-D-ribo-pentofuranosyl)cytosine (ECyd), Is a Potent Inhibitor of RNA Synthesis," Nucleosides Nucleotides, Vol. 18, No. 4-5, 1999, pp. 811-814. doi:10.1080/15257779908041568

[26] A. Azuma, A. Matsuda, T. Sasaki and M. Fukushima, "1(3-C-ethynyl- $\beta$-D-ribo-pentofuranosyl)cytosine (ECyd, TAS-106)1: Antitumor Effect and Mechanism of Action," Nucleosides Nucleotides Nucleic Acids, Vol. 20, No. 4-7, 2001, pp. 609-619. doi:10.1081/NCN-100002337

[27] A. Matsuda and T. Sasaki, "Antitumor Activity of SugarModified Cytosine Nucleosides," Cancer Science, Vol. 95, No. 2, 2004, pp. 105-111. doi:10.1111/j.1349-7006.2004.tb03189.x

[28] D. Murata, Y. Endo, T. Obata, K. Sakamoto, Y. Syouji, M. Kadohira, A. Matsuda and T. Sasaki, "A Crucial Role of Uridine/Cytidine Kinase 2 in Antitumor Activity of 3'Ethynyl Nucleosides," Drug Metabolism and Disposition, Vol. 32, No. 10, 2004, pp. 1178-1182. doi:10.1124/dmd.104.000737 